\title{
A Four-Element Sensor Array Consisting of Asymmetric Distributed-Feedback Fiber Lasers
}

\author{
Pengpeng WANG ${ }^{1}$, Jun $\mathrm{CHANG}^{1 *}$, Cunguang $\mathrm{ZHU}^{1}$, Boning SUN ${ }^{1}$, \\ Guangping $\mathrm{LV}^{1}$, Sasa ZHANG ${ }^{1}$, Yanjie ZHAO ${ }^{1,2}$, Zhihui SUN ${ }^{2}$, \\ Xiaolei ZHANG ${ }^{2}$, and Gangding PENG ${ }^{1,2,3}$
}

\author{
${ }^{1}$ School of Information Science and Engineering and Shandong Provincial Key Laboratory of Laser Technology and \\ Application, Shandong University, Jinan, 250100, China \\ ${ }^{2}$ Laser Institute of Shandong Academy of Science, Jinan, 250014, China \\ ${ }^{3}$ Photonics \& Optical Communications Group, School of Electrical Engineering \& Telecommunications, the University \\ of New South Wales, Sydney 2052, Australia \\ *Corresponding author: Jun CHANGＥ-mail: changjun@sdu.edu.cn
}

\begin{abstract}
A sensor array system formed by arranging four asymmetric distributed-feedback fiber lasers (DFB-FL) in ascending order according to their slope efficiencies was proposed. The output flatness could be effectively improved with the application of asymmetric DFB-FLs. The last element had almost the same output with the others although it obtained the smallest pump power. The relative intensity noise (RIN) and relaxation oscillation frequency of the sensor array were also analyzed. It is found that the relaxation oscillation frequency of a certain DFB-FL was relevant to its relative position in the array. And the RIN of a certain DFB-FL was always affected by the other elements in the array, which was not dependent on the order of their arrangement.
\end{abstract}

Keywords: Asymmetric DFB fiber laser, sensor array, intensity noise, relaxation oscillation

Citation: Pengpeng WANG, Jun CHANG, Cunguang ZHU, Boning SUN, Guangping LV, Sasa ZHANG, et al., "A Four-Element Sensor Array Consisting of Asymmetric Distributed-Feedback Fiber Lasers," Photonic Sensors, 2014, 4(2): 180-187.

\section{Introduction}

In recent years, many attractive features of distributed-feedback fiber laser (DFB-FL) have been reported with regard to their applications in sensing systems [1-4]. The DFB-FL exhibits great advantages including the small size, simplicity, extremely high sensitivity, intrinsically narrow emission line width, robust signal mode operation, remote pumping, and inherent high multiplexing capability. Owing to the intrinsically narrow emission line width and low frequency noise level of the DFB-FL, it is potential to achieve the ultra-high detection resolution by means of interferometry [5]. Another attractive feature of the DFB-FL is the high multiplexing capability. Integrating a wavelengthdivision multiplexing (WDM) array into a single fiber has particular strengths in construction of an ultrathin sensing array [6, 7]. A desirable configuration of the DFB-FL sensor array system would consist of a series of short cavity DFB-FLs at discrete wavelengths, which are multiplexed together according to the WDM scheme, and collinearly pumped by a single pump laser. The

Received: 14 December 2013 / Revised version: 28 December 2013

(C) The Author(s) 2014. This article is published with open access at Springerlink.com

DOI: $10.1007 / \mathrm{s} 13320-014-0167-2$

Article type: Regular 
outputs are interrogated by a single read-out interferometer, and each laser signal is demultiplexed by a dense wavelength division demultiplexor (DWDM) [8,9].

In a sensor array, a most important issue is the pump power budget. Various losses exist in the optical transmission path, such as the pump absorption and transmission losses, splice loss, and passive component insertion loss. These issues limit the number of elements in the sensor array and determine the pump power distribution directly. Some researchers have investigated it in detail [10], shown as Fig. 1, which is quoted from [10] Fig. 6-3. The magnitudes of all kinds of losses are revealed, and the maximum transmission distance of the pump power is shown. It can be obviously observed that the pump power for the DFB-FLs decreases in series. Therefore, the last DFB-FL of the sensor array always obtains the smallest pump power, which may not approach the pump threshold and has no laser emitting. And also, the output flatness problem could be caused if the DFB-FLs in the array have the same output slope efficiency. So some DFB-FLs with the high output should be chosen, and the DFB-FL with the higher slope efficiency could be used as the last element. In this paper, asymmetric DFB-FLs are used. A very desirable feature of asymmetric DFB-FL is unidirectionality $[11,12]$. By placing the phase shift asymmetrically with respect to the grating center, the lager output power could be obtained from the shorter end. Along with the other parameters, such as and coupling coefficient $\kappa$, asymmetric DFB-FLs with different slope efficiencies would be fabricated. In this paper, the structure design and operation characteristics of the asymmetric DFB-FLs are introduced, and then a four-element sensor array is formed by arranging the four DFB-FLs in ascending order according to their slope efficiencies.

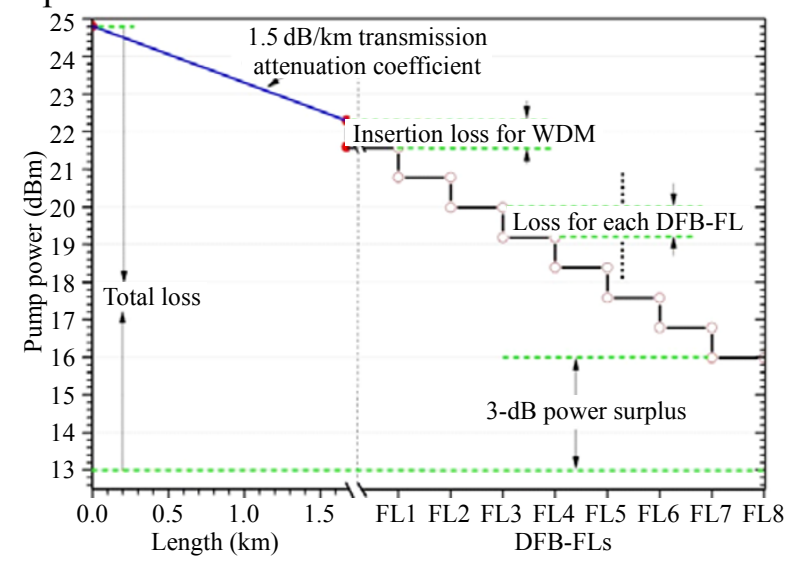

Fig. 1 Pump power budget of the sensor array.

The DFB-FL intensity noise plays an important role in determining the minimum detectable signal $[13,14]$, and the minimum detectable signal of each wavelength element determinates the viability of the DFB-FL sensor array [6]. A number of groups have investigated the intensity noise characteristics of the DFB-FL and obtained some meaningful conclusions. Cranch et al. have modeled the intensity noise of $\mathrm{Er}^{3+}$ doped DFB-FL according to the rate equations [14]. In a sensor array, the intensity noise is becoming more important and sophisticated. Because there exists not only various losses, but external injected lasers from other elements are generated, which would finally be transfered into the laser intensity noise. Lina Ma et al. have modeled the relative intensity noise (RIN) of the DFB-FL with external laser injection, the equation is shown as (1), and the RIN enhancement for each sensing element in the array could be evaluated exactly $[15,16]$.

$$
R I N(f)=\left|H_{P}(f)\right|^{2} \frac{\delta W_{P}(f)^{2}}{W_{P 0}{ }^{2}}+\left|H_{L}(f)\right|^{2} \frac{\delta \gamma(f)^{2}}{\gamma_{0}{ }^{2}}+\left|H_{e}(f)\right|^{2} \frac{\delta Q^{\prime}(f)}{q_{0}^{\prime}}
$$

where $H_{P}(f), H_{L}(f)$, and $H_{e}(f)$ are the transfer functions for pump power fluctuation, cavity loss modulation, and injected power perturbation, respectively. The last term expresses the RIN induced by external laser injection. The relaxation oscillation frequency also plays an important role in the investigation into the sensor array. Because the relationship between the relaxation oscillation 
frequency and actual absorbed pump power is not affected by external laser injection [15, 16], the actual pump power for one element in a sensor array can be inferred by calibrating the relaxation oscillation frequency. In this paper, the intensity noise and relaxation oscillation characteristics of the sensor array are also investigated in detail by experiments. When we built the experimental platform, we have tried our best to minimize the splice losses and the Rayleigh scattering effect etc. And then the RIN and relaxation oscillation of a certain DFB-FL with the other DFB-FLs laser injections would be measured and compared with its actual values.

\section{Asymmetric DFB-FL design}

The asymmetric DFB-FL was designed by placing the $\pi$ phase shift asymmetrically with respect to the grating center, as shown in Fig.2. In this design, the refractive index modulation $\Delta n$ was distributed inside the DFB-FL with a uniform profile. Each grating segment on either side of the $\pi$ phase shift could be considered as a separate reflector, $M_{1}$ and $M_{2}$, as shown in Fig. 2. The reflectivity $R$ of a grating with the constant gain at the Bragg wavelength is given by [11]

$$
R=|r|^{2} \approx \tanh ^{2}(k L) .
$$

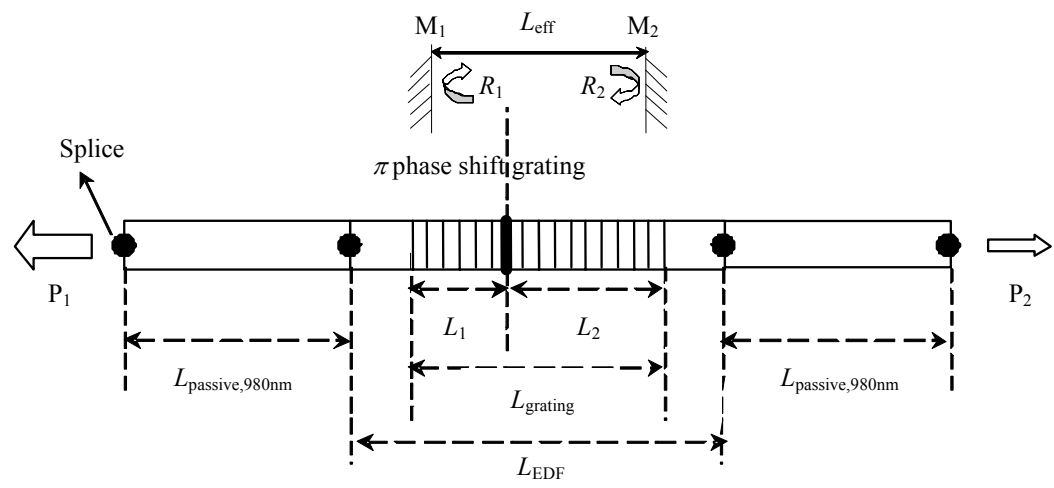

Fig. 2 Asymmetric $\pi$ phase shift design and unidirectional output operation.

For this asymmetric DFB-FL, the coupling coefficient $\kappa$, which is shown as $\kappa=\pi \Delta n / \lambda_{B}$, is considered as the constant. By moving the $\pi$ phase shift, we can change the lengths of the left-hand grating $L_{1}$ and right-hand grating $L_{2}$, which leads to the change in the effective reflectivity $R_{1}$ and $R_{2}$. When the $\pi$ phase shift is located in the middle of the grating, the cavity is symmetric. Thus, not only the reflectivities $R_{1}$ and $R_{2}$ but also the output powers at both ends are equal. When the $\pi$ phase shift is placed asymmetrically with respect to the grating center, the output powers at both ends become unequal, and the larger output power can be obtained from the shorter end. Figure 3(a) in [11] shows the variation of the shorter end output power with the position of the $\pi$ phase shift $Z_{\pi}$ for three different $\kappa$ values. It is shown that there is an optimum $Z_{\pi}$ that results in an overall maximum output power. It is also evident that for each $Z_{\pi}$ there is a different optimum $\kappa$ value that results in the maximum output power from the desired end.

In our laboratory, four asymmetric DFB-FLs with different wavelengths were fabricated by the phase mask moving method. All of them were designed by placing the $\pi$ phase shift at the position $Z_{\pi}=0.4 L_{\text {grating, }}$, and $L_{\text {grating }}$ was the $\pi$ phase shift grating length. For DFB-FL1, $L_{\text {grating }}=0.045 \mathrm{~m}$, and for the others, $L_{\text {grating }}=0.04 \mathrm{~m}$. The length of the erbium doped fiber amplifier (EDF), $L_{\mathrm{EDF}}$, was $0.05 \mathrm{~m}$. The passive fibers used at both ends were $980 \mathrm{~nm}$ transmission fibers, and $L_{\text {passive }}=0.5 \mathrm{~m}$. The coupling coefficient $\kappa$ values were set in the range of $120 \mathrm{~m}^{-1}-150 \mathrm{~m}^{-1}$. All the different fibers were spliced directly, and the splice loss was controlled within $0.01 \mathrm{~dB}$.

The characteristics of all DFB-FLs were 
measured separately before they were utilized to construct a sensor array. Output powers at both ends of DFB-FLs were measured and presented in Fig. 3. Larger output powers were obtained from the shorter ends $\left(\mathrm{P}_{1}\right)$. The output powers of the longer ends $\left(\mathrm{P}_{2}\right)$ were so small that almost could be ignored. Taking DFB-FL4 for instance, after linear fitting, the slope efficiency of $\mathrm{P}_{1}$ was 0.6829 , the slope efficiency of $\mathrm{P}_{2}$ was 0.00939 , and their ratio was 72.73 . The unidirectional output power was easily achieved by this asymmetric design. In our experiments, we only considered the shorter end $\left(\mathrm{P}_{1}\right)$ output power. As shown in Fig. 3, the output powers of all the four DFB-FLs increased linearly with an increase in the 1480-nm pump power. The threshold pump powers of the four DFB-FLs were discovered in the range of $5 \mathrm{~mW}-10 \mathrm{~mW}$. The DFB-FL with the largest slope efficiency had the smallest threshold. Owing to different grating lengths and coupling coefficients, the slope efficiencies of the four DFB-FLs were different. Therefore, the DFB-FL with the larger slope efficiency can be used as the rear element of the sensor array.

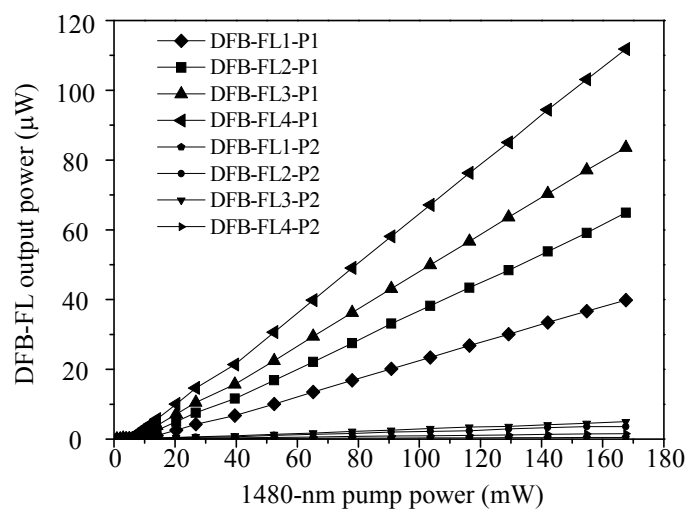

Fig. 3 Output powers at both ends of DFB-FLs.

The RIN at $10 \mathrm{kHz}$ of the four DFB-FLs were measured and are shown in Fig. 4(a). Their changing trends with the increasing pump power were virtually identical. And the RIN characteristics of DFB-FL1 and DFB-FL3 versus the 1480-nm pump power looked basically the same. Relaxation oscillation frequencies of the four DFB-FLs are given in Fig. 4(b). They all increased with an increase in the pump power and had almost the same changing trends. For a certain pump power, DFB-FL1 had the smallest relaxation oscillation frequency, and DFB-FL2 and DFB-FL4 had nearly the identical ones.

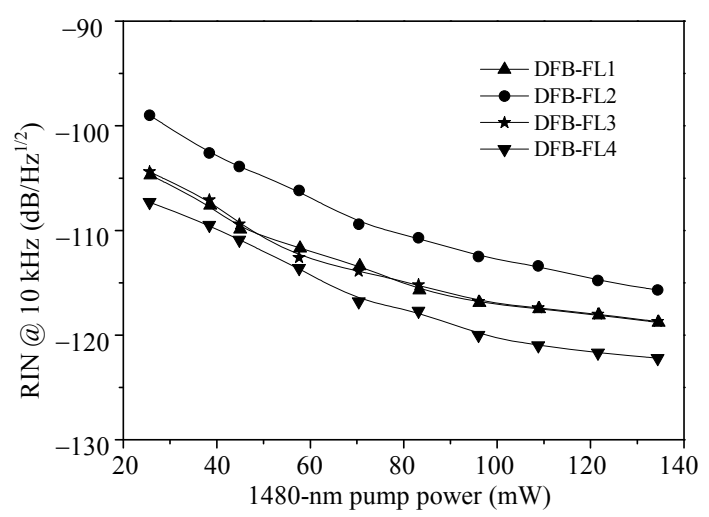

(a) RIN of DFB-FLs at $10 \mathrm{kHz}$

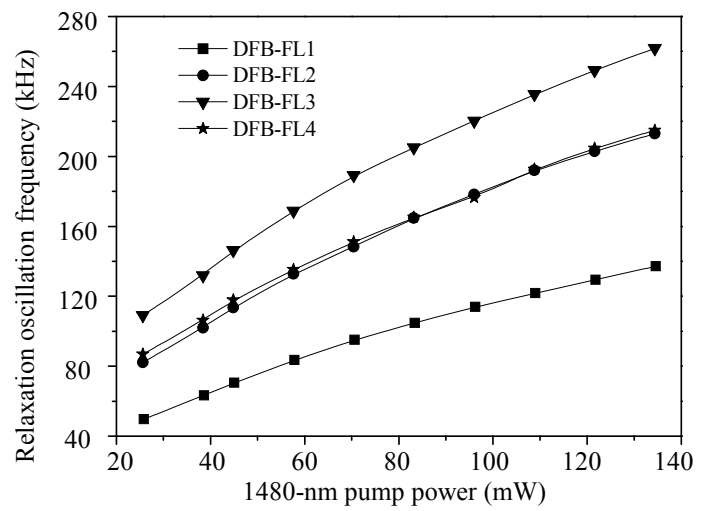

(b) Relaxation oscillation frequencies of DFB-FLs

Fig. 4 RIN and relaxation oscillation frequencies of DFB-FLs.

\section{Experiments and results}

Figure 5 shows the experimental setup. Four DFB-FLs were arrayed according to their slope efficiencies in ascending order. In this sensor array, the fibers were all spliced together directly, and the splice losses were controlled within $0.01 \mathrm{~dB}$. Including the 1480-nm pump and WDM splice core, WDM and optical isolator (ISO) splice cores, there were 15 splice cores in the light transmission path. Rayleigh scattering is another important issue which may create instability in the sensor array, and the critical length of each DFB-FL was calculated [10]. The smallest critical length was $30 \mathrm{~m}$. The length of the sensor array was less than $6 \mathrm{~m}$, and the stability 
of the sensor array would not be affected by the Rayleigh scattering effect. And at the end of the sensor array, a circle with the $1-\mathrm{cm}$ diameter was made in order to reduce the fiber end reflection effect. Each laser was designed to operate at a different wavelength: DFB-FL1 was $1541.09 \mathrm{~nm}$, DFB-FL2 was $1530.27 \mathrm{~nm}$, DFB-FL3 was $1532.72 \mathrm{~nm}$, and DFB-FL4 was $1539.15 \mathrm{~nm}$. The lasers were pumped by a $1480-\mathrm{nm}$ semiconductor laser source via a 1480/1550 WDM coupler. An optical isolator was inserted to suppress the feedback induced noise in the system.

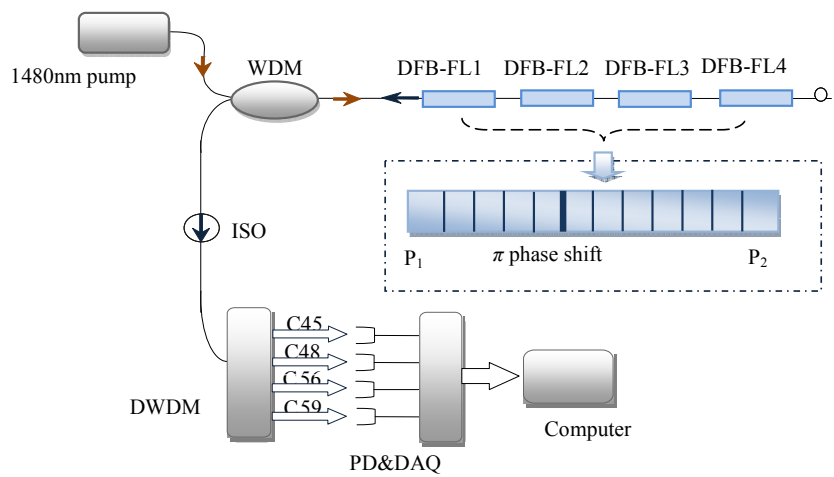

Fig. 5 Experimental setup.

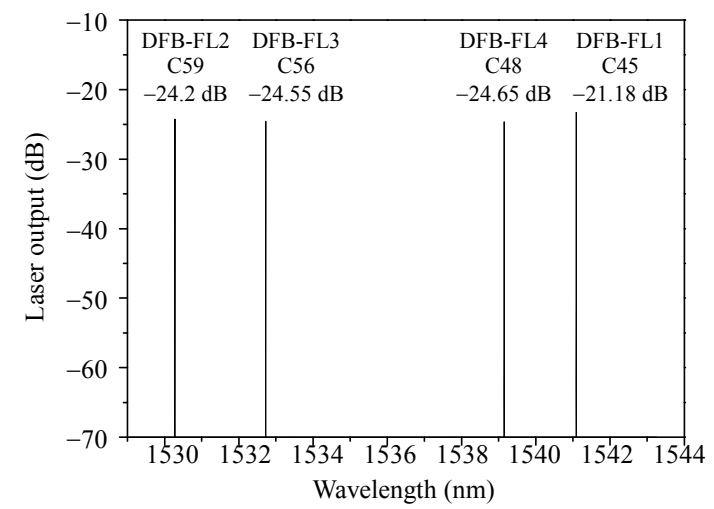

Fig. 6 Optical spectrum analyzer output of the four-element sensor array.

In our experiments, the optical spectrum was measured firstly. The laser output from the ISO was directly input into the optical spectrum analyzer (OSA) (AP2040A APEX France) with a spectral resolution of $0.16 \mathrm{pm}$. Through experiment, we found that the threshold of the sensor array was $11.7 \mathrm{~mW}$, and DFB-FL2 was the last to emit laser. Figure 6 shows the optical spectrum of the sensor array when the pump power was $185.6 \mathrm{~mW}$. We could obviously see that the first element, DFB-FL1 $(1541.09 \mathrm{~nm})$, had the highest amplitude, the other three had mostly the same amplitudes, and the power equilibration among them was less than $0.5 \mathrm{~dB}$. This is because DFB-FL1 was most close to the 1480-nm pump source, and the other DFB-FLs at the back obtained the lower pump powers due to the absorption of the front DFB-FLs. As expected, a stable and flat output was obtained. It confirms that the asymmetric DFB-FL has the great potential for improving the power equilibration problem. The last element had a higher output although it obtained the smallest pump power.

Then, the RIN and relaxation oscillation of the sensor array were investigated in detail under this premise. As shown in Fig. 5, a DWDM was used to split off the individual element signals as a wavelength filter. Elements were set at the corresponding element from the International Telecommunications Union (ITU) grid. Each element of the desired signal was detected by a low-noise photodiode and an amplifier circuit. The analog output of the photo-detector was sampled by an acquisition card, and a fast Fourier transform process was completed through a software program. Then, the RIN spectrum could be displayed by the computer. DFB-FL1 and DFB-FL4 were chosen as the test objects. For DFB-FL1, when the 1480-nm pump power was $95.5 \mathrm{~mW}$, the RIN and relaxation oscillation frequency were measured. Then, we connected DFB-FL2 at the back of DFB-FL1 and used the DWDM to measure the RIN spectrum of DFB-FL1 again. By such analogy, DFB-FL3 was connected after DFB-FL2 and DFB-FL4 was connected after DFB-FL3. Thus, the RIN and relaxation oscillation frequency of DFB-FL1 with the other DFB-FLs laser injections were obtained. Similarly, the RIN and relaxation oscillation frequency of DFB-FL4 with the others laser injections were measured. The 1480-nm pump power remained the same. The only difference is that the other DFB-FLs were connected in the front 
in series. All the measured results are summarized in Table 1. Obviously, the relaxation oscillation frequency $\left(f_{\text {ro }}\right)$ of DFB-FL1 had the slight change, which could be virtually ignored. The RIN of DFB-FL1 at $10 \mathrm{kHz}$ increased continually when DFB-FL2, DFB-FL3, and DFB-FL4 were connected in series. Comparing the measured results of DFB-FL4, we can find that the relaxation oscillation frequency decreased, and the RIN at $10 \mathrm{kHz}$ increased continually.

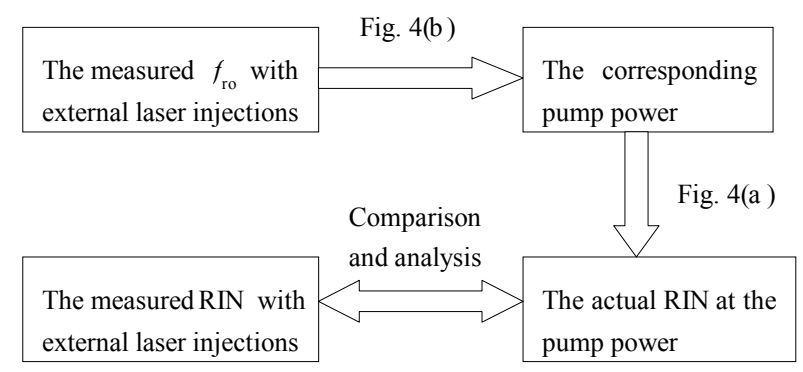

Fig. 7 Analysis process.

The analysis process is shown in Fig. 7. By using

Table 1 Characteristics of DFB-FL1 and DFB-FL4. frequencies, the corresponding pump powers were obtained and are given in Table 1. Comparing the pump powers of DFB-FL1 and DFB-FL4, differences could be directly observed. The pump power of DFB-FL1 was close to the pump power added to the array and changed slightly no matter how many DFB-FLs were connected at its back. But the pump power of DFB-FL4 decreased continually when other DFB-FLs were connected in its front in series. The reason is that the pump power was not completely absorbed by DFB-FL1, and the rest of the power would be transmitted into the next element. It is fair to say that the first element had a priority to get the pump power and could not be affected by the other DFB-FLs which were connected to its back. By contrast, the pump power of DFB-FL4 was seriously affected by the DFB-FLs which were connected to its front. The more elements the sensor array had, the smaller pump power the last element could obtain.

\begin{tabular}{cccc||cccc}
\hline DFB-FL & RIN @10kHz $\left(\mathrm{dB} / \mathrm{Hz}^{1 / 2}\right)$ & $f_{r o}(\mathrm{kHz})$ & Pump power $(\mathrm{mW})$ & DFB-FL & RIN @10kHz $\left(\mathrm{dB} / \mathrm{Hz}^{1 / 2}\right)$ & $f_{\text {ro }}(\mathrm{kHz})$ & Pump power $(\mathrm{mW})$ \\
\hline FL1 & -116.5 & 113.043 & 93.5 & FL4 & -119.5 & 175.985 & 94.0 \\
FL1(FL2) & -114.3 & 110.628 & 92.1 & (FL1)FL4 & -112 & 131.401 & 54.5 \\
FL1(FL2,FL3) & -112.1 & 111.094 & 92.6 & (FL1,FL2)FL4 & -109.4 & 123.671 & 48.7 \\
FL1(FL2,FL3,FL4) & -110.6 & 110.628 & 92.1 & (FL1,FL2,FL3)FL4 & -107.1 & 112.560 & 41.5 \\
\hline
\end{tabular}

The actual RIN and the measured RIN with different external laser injections of DFB-FL1 and DFB-FL4 were obtained and are shown in Fig. 8.

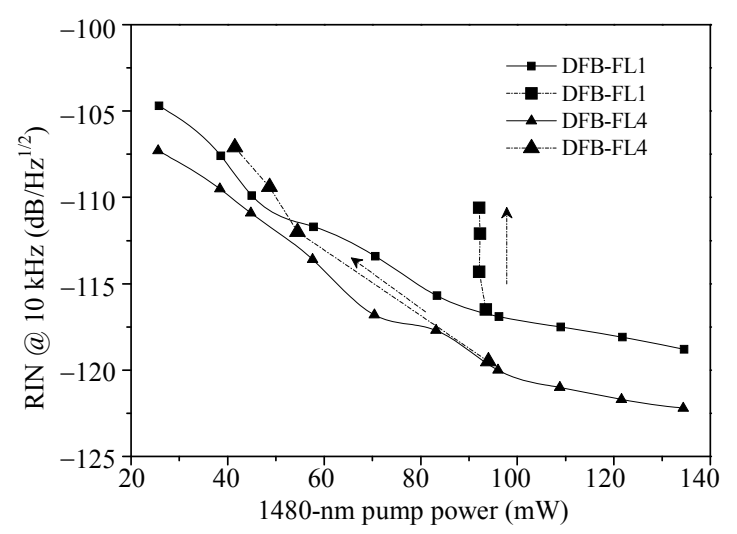

Fig. 8 Comparing results (dot lines are RIN with external laser injections and solid lines are actual RIN).
The symbols on the dot lines are the RIN with different external laser injections. The solid lines are the actual RIN. Through comparison, we find that the RIN of DFB-FL1 at $10 \mathrm{kHz}$ increased from $-116.5 \mathrm{~dB} / \mathrm{Hz}^{1 / 2}$ to $-110.6 \mathrm{~dB} / \mathrm{Hz}^{1 / 2}$ step by step when the other three DFB-FLs were connected to its back in series, as indicated by the arrow. The triangular symbols on the dot line also show that the RIN of DFB-FL4 increased constantly when the other three DFB-FLs were connected to its front in series. For instance, when the pump power was $41.5 \mathrm{~mW}$, the actual RIN at $10 \mathrm{kHz}$ of DFB-FL4 was $-110.1 \mathrm{~dB} / \mathrm{Hz}^{1 / 2}$. But the RIN increased to $-107.1 \mathrm{~dB} / \mathrm{Hz}^{1 / 2}$ when the other three were all connected. It confirms that the RIN of DFB-FL of 
any location would be always affected by the other DFB-FLs. The RIN of the first element had a larger increase. The reason for this difference should be that the output powers at different ends of the asymmetric DFB-FL were unequal, and much smaller laser which came from Port $\mathrm{P}_{2}$ was transmitted into the rear DFB-FL.

\section{Conclusions}

In this paper, a four-element sensor array system consisted of asymmetric DFB-FLs is introduced. The asymmetric DFB-FLs were fabricated by the phase mask moving method, and their structure design and operation characteristics are presented. The four DFB-FLs were arrayed according to their slope efficiencies in ascending order. The optical spectrum of the sensor array shows that the first element had the highest output power, and the other three had substantially the same output power. The power equilibration among the rear three DFB-FLs was less than $0.5 \mathrm{~dB}$. The last element had a higher output although it obtained the smallest pump power. The application of asymmetric DFB-FLs could effectively improve the output flatness. As expected, a stable output of the sensor array was achieved. We experimentally demonstrated that the relaxation oscillation frequency of DFB-FL1 barely changed when the other DFB-FLs were connected to its back end in series, and the relaxation oscillation frequency of DFB-FL4 changed a lot when the others were connected to its front end in series, which is because the first element had a priority to obtain the pump power. The pump power was absorbed by the element in the front, and the rest would be transmitted into the next element and absorbed. So the pump power of the first element could not be affected by the other DFB-FLs which were connected to its back. By contrast, the pump power of the last element was seriously affected by the DFB-FLs which were connected to its front. The more elements the sensor array had, the smaller pump power the last element could obtain. Unlike the relaxation oscillation frequency, the RIN of DFB-FL was always affected by the other DFB-FLs no matter how they were arranged. The only difference was the values which were added on the RIN. The RIN of the rear element had a smaller increase. The reason for this result should be that the output powers at different ends of the asymmetric DFB-FL were unequal, and much smaller laser (Port $\mathrm{P}_{2}$ ) was transmitted into the rear DFB-FL. It confirms that the RIN induced by the front external laser injections could be effectively reduced by using DFB-FLs.

\section{Acknowledgment}

This work was supported by the Natural Science Foundation of China (60977058\& 61205083), Independent Innovation Foundation of Shandong University (IIFSDU2010JC002 \&2012JC015) and the key technology projects of Shandong Province (2010GGX10137).

Open Access This article is distributed under the terms of the Creative Commons Attribution License which permits any use, distribution, and reproduction in any medium, provided the original author(s) and source are credited.

\section{References}

[1] G. A. Cranch, G. M. H. Flockhart, and C. K. Kirkendall, "Distributed feedback fiber laser strain sensors," IEEE Sensors Journal, 2008, 8(7): 1161-1172.

[2] S. Foster, A. Tikhomirov, and J. Velzen, "Towards a high performance fiber laser hydrophone," Journal of Lightwave Technology, 2011, 29, 1335-1342.

[3] T. Xu, F. Li, and Y. Liu, "Characteristics of frequency noise in a fiber laser sensor array," in Proc. SPIE (International Conference on Optical Instruments and Technology: Advanced Sensor Technologies and Applications, Beijing), vol. 7157, pp. 71571A1 -71571A 5, 2008.

[4] I. Azmi, I. Leung, X. B. Chen, S. L. Zhou, Q. Zhu, K. Gao, et al., "Fiber laser based hydrophone systems," Photonic Sensors, 2011, 1(3): 210-221.

[5] K. P. Koo and A. D. Kersey, "Fibre laser sensor with ultrahigh strain resolution using interferometric interrogation," Electronics Letters, 1995, 31(14): 
1180-1182.

[6] K. P. Koo and A. D. Kersey, "Noise and cross talk of a 4-element serial fiber laser sensor array," Optical Fiber Communications, OFC'96, pp. 266-267, 1996.

[7] G. A. Cranch, S. Foster, and C. K. Kirkendall, "Fiber laser strain sensors: enabling a new generation of miniaturized high performance sensors," in Proc. SPTE (20th International Conference on Optical Fiber Sensors), Edinburgh, vol. 7503, pp. 834331, 2009.

[8] S. Foster, A. Tikhomirov, M. Englund, H. Inglis, G. Edvell, and M. Milnes, "A 16 element fibre laser sensor array," in the 18th Internetional Conference Optical Fiber Technology, Australia, pp. 40-42, 2006.

[9] Y. Jiang, "Wavelength division multiplexing addressed four-element fiber optical laser hydrophone array," Applied Optics, 2007, 46(15): 2939-2948.

[10] W. Tuan, "Research on distributed feedback fiber lasers," Ph.D. dissertation, Institute of Semiconductors Chinese Academy of Sciences, China, 2010.

[11] K. Yelen, L. M. B. Hickey, and M. N. Zervas, “A new design approach for fiber DFB lasers with improved efficiency," IEEE Journal of Quantum Electron, 2004, 40(6): 711-720.

[12] K. Yelen, M. N. Zervas, and L. M. B. Hickey, "Fiber DFB lasers with ultimate efficiency," Journal of Lightwave Technology, 2005, 23(1): 32-43.

[13] S. Foster, A. Tikhomirov, and M. Milnes, "Fundamental thermal noise in Distributed feedback fiber lasers," IEEE Journal of Quantum Electronics, 2007, 43(5): 378-384.

[14] G. A. Cranch, M. A. Englund, and C. K. Kirkendall, "Intensity noise characteristics of Erbium-doped distributed-feedback fiber lasers," IEEE Journal of Quantum Electronics, 2003, 39(12): 1579-1586.

[15] L. N. Ma, Y. M. Hu, S. D. Xiong, Z. Meng, and Z. L. $\mathrm{Hu}$, "Intensity noise and relaxation oscillation of a fiber laser senor array integrated in a single fiber," Optics Letters, 2010, 35(11): 1795-1797.

[16] L. Ma, "Fiber laser hydrophone," Ph.D. dissertation, Graduate School of National University of Defense Technology, China, 2010.

[17] E. Ronnekleiv, "Frequency and intensity noise of single frequency fiber Bragg grating lasers," Optical Fiber Technology, 7(3): 206-235, 2001. 